\title{
Generation of Antibodies Against Rice stripe virus Proteins Based on Recombinant Proteins and Synthetic Polypeptides
}

\author{
Sen Lian ${ }^{1 \dagger}$, Miranda Gilda Jonson ${ }^{1,2}$, Won Kyong Cho ${ }^{1}$, Hong-Soo Choi ${ }^{3}$, Yeon-Ho Je ${ }^{1}$ and Kook-Hyung Kim ${ }^{1 *}$ \\ ${ }^{1}$ Department of Agricultural Biotechnology and Plant Genomics and Breeding Institute, Seoul National University, Seoul 151- \\ 921, Korea \\ ${ }^{2}$ Department of Biology, School of Science and Engineering, Ateneo de Manila University, Katipunan Avenue, Loyola Heights \\ Quezon City 1108, Philippines \\ ${ }^{3}$ Department of Agricultural Biology, National Academy of Agricultural Science, RDA, Suwon 441-707, Korea
}

(Received on November 5, 2010; Accepted on January 23, 2011)

\begin{abstract}
Rice stripe virus (RSV) is one of serious epidemic pathogens for rice species grown in many Asian countries. Therefore, it is necessary to produce a diagnostic detection kit applicable in fields for RSV detection. In this study, RSV proteins that were derived from recombinant proteins and synthetic polypeptides as antigens were generated and were raised in rabbits for antiserum production. Among seven proteins in RSV, genes that code for NCP and NS3 proteins were cloned and subcloned into vector carrying His-tag protein and were expressed in E. coli. Of two recombinant proteins, only anti-NCP displayed stable hybridization signals in western blot analysis. Alternately, synthetic RSV polypeptides for CP, NCP, NS3 and NSve4 we also generated and only antibodies against $\mathrm{CP}$ and NCP were very effective to detect RSV in both RSV infected rice and weed plants. However, antibodies against NS3 and NSve4 showed weak specific bands as well as strong non-specific background due to the difference of viral proteins produced in the infected leaves. In summary, the antibodies generated against RSV proteins produced in this study will be useful for various assays such as for RSV diagnostic detection, immunoprecipitation, protein purification, and western blot analysis.
\end{abstract}

Keywords : antibody, NCP, rice, rice stripe virus

Rice (Oryza sativa) is one of most important crops in the world, especially, in Eastern Asia. Of known pathogens infecting rice, the rice stripe disease caused by Rice stripe virus (RSV) lead to dramatic hazardous impact on rice quantity and quality. RSV, a member of the genus Tenuivirus, has known to be one of economically important virus with

\footnotetext{
*Corresponding author.

Phone)+82-2-880-4677, FAX) +82-2-873-2317

E-mail) kookkim@snu.ac.kr

${ }^{\dagger} E$ Equally contributed.
}

severe symptoms such as chlorotic stripes, chlorosis, and necrosis in leaves of many cultivated rice varieties (Hibino, 1996; Toriyama, 2000). Interestingly, most of Japanese low land rice varieties are highly susceptible to RSV while Japanese upland rice varieties and Indica rice varieties are resistant or tolerant to RSV (Maeda et al., 2006).

Since the first report of RSV in 1965 in Korea, the RSV disease is gradually regarded as a severe disease (Toriyama, 2000), and had become a very important virus disease during 2007-2009. Also, in these period that RSV caused serious damage on rice production affecting $84 \%$ of total paddy field in many areas (Choi and Kim, unpublished). RSV occurred in temperate countries such as China and Japan (Abo and Sy, 1998; Hibino, 1996). RSV can also infect not only rice but also plant species belonging to the family Gramineae including maize, wheat, oat, foxtail millet, and several weeds (Toriyama, 2000). This virus is transmitted by mostly Laodelphax striatellus as well as three other plant hoppers.

The morphology of RSV particles are thin and filamentous shape without envelope. RSV is composed of four single stranded RNA segments named as RNA1, 2, 3, and 4 based on their segment size (Hamamatsu et al., 1993). RNA1 ( $\sim 8.9 \mathrm{~kb})$ is the largest RNA segment which encodes for RNA-dependent RNA polymerase (RdRp) protein in negative sense orientation. In contrast, each of RNA2, 3, and 4, encodes two proteins, respectively, in ambisense orientation. RNA2 codes for NS2 (unknown function) and NSvc2 (glycoprotein). NS3 and CP proteins are encoded by RNA3 whereas NCP (disease specific protein) and NSvc4 (movement protein) proteins are encoded by RNA4 (Xiong et al., 2008, 2009).

Recent phylogenetic studies have shown that RSV isolates in Korea showed mixtures of RSV populations from China and Japan suggesting that there was a possible occurrence of natural genetic reassortment of RSV segments from these countries (Jonson et al., 2009a; 2009b). 
Moreover, the RSV populations in China also displayed high divergence caused by frequent gene flow, however, it seems that there is no invasion of new RSV subtypes in China (Wei et al., 2009).

The generation of antibodies against RSV proteins can be practically used in a wide range of application. First, they can be used to detect RSV in both RSV-infected rice plants and weeds as well as the viruliferous brown planthoppers by western blot and enzyme-linked immunosorbent assay (ELISA). Secondly, the localization of RSV in rice plants can be also observed by immunofluorescent or immunogold microscopy using the viral antibodies. Thirdly, it is also possible to find host factors that interact with viral proteins by immunoprecipitation.

In this study, we developed antibodies for RSV proteins using recombinant proteins and synthetic polypeptides used as antigens. To test the obtained antibodies for RSV detection, we performed western blot analysis using several RSV infected plants including rice and weeds.

\section{Materials and Methods}

RNA isolation from RSV infected plants. The total RNA was extracted from RSV infected plants following procedures as previously described (Jonson et al., 2009a, 2009b). The isolated total RNA was stored at $-20^{\circ} \mathrm{C}$ for further study.

Viral gene amplification and cloning to construct recombinant plasmid. We have amplified $N C P(536 \mathrm{bp})$ and NS3 (636 bp) genes of RSV by RT-PCR using specific primers designed from previously obtained complete nucleotide sequences (Jonson et al., 2009a, 2009b). Each primer containing EcoRI and HindIII restriction sites is as follows: NCP for 5-AGA GAA TTC ATG CAA GAC GTA CAA AGG AC-3, NCP rev 5-TCT AAG CTT CTA TGT NTT GTG TAG AAG Ä-3, NS3 for 5-AGA GAA TTC ATG AAC GTG TTC ACA TCG TC-3, and NS3 rev 5-TCT AAG CTT CTA CAG CAC AGT GGA GAG C-3. The amplified PCR products were purified and cloned into pGEM-T easy vector (Promega, Madison, USA). Cloned plasmids were transformed into $E$. coli $\mathrm{DH} 10 \mathrm{~B}$. For the production of viral proteins fused with His-tag, DNA fragments for NCP and NS3 in pGEM-T easy vector were digested by EcoRI and HindIII enzymes (New England Biolabs, Ipswich, UK) and then were ligated into pET-28 and pET-30 expression vectors carrying His-tag at Nterminal region, respectively. His-tag fused proteins were expressed in E. coli BL21 and were purified according to manufacturer's instruction (Merck, Darmstadt, Germany).

Expression and purification of His-tag fusion proteins. Each cloned pET-28 or pET-30 plasmid was transformed into E. coli BL21 (DE3). An overnight culture of transformed $E$. coli was diluted 1:100 in fresh LB (LuriaBertani) medium and incubated at $30{ }^{\circ} \mathrm{C}$ until the $\mathrm{OD}_{600}$ value reached 0.6. Expression of His-tagged RSV NCP and NS3 were induced with $1 \mathrm{mM}$ isopropyl- $\beta$-D-thiogalactopyranoside (IPTG) for 2 hours at $30^{\circ} \mathrm{C}$. The cells were immediately chilled on ice, collected by centrifugation at $10,000 \mathrm{rpm}$ for $5 \mathrm{~min}$ using Supra $25 \mathrm{~K}$ centrifuge (Hanil Science, Incheon, Korea), and pellet was frozen at $-70{ }^{\circ} \mathrm{C}$, thawed, resuspended in extraction buffer $(20 \mathrm{mM}$ sodium phosphate, $500 \mathrm{mM} \mathrm{NaCl}, 10 \mathrm{mM}$ Imidazole $\mathrm{pH}$ 7.4), and disrupted by sonication on ice. Cell lysate was centrifuged at 13,000 rpm for 20 min using Supra $25 \mathrm{~K}$ centrifuge and the recombinant protein was purified from the soluble fraction by affinity chromatography using a Ni/NTA column (QIAGEN, Hilden, Germany). Purified proteins were first analyzed by SDS-PAGE (Sodium Dodecyl Sulfate Polyacrylamide Gel electrophoresis).

Polypeptide design for antibody production. To select proper polypeptides for RSV protein antibody production, several peptides were designed by AbFrontier company (Young In Frontier, Seoul, Korea). For antibody production, optimal length of peptides is 15-25 amino acids (aa). Longer peptides more than 25 aa can be used with high cost and short peptides less than 10 aa may present limited number of epitope (http://www.anaspec.com/html/antibody_notes.html). As a result, several polypeptides up to six were designed (Table 2). Among five or six peptides for each target protein, two peptides which can most likely accessible to the full length protein were selected based on a prediction program and expertise. Antibody was produced by coimmunizing with two selected peptides.

Antibody production. For the production of polyclonal antibodies, recombinant proteins were used as antigens for NCP and NS3, and that synthetic peptides were used for NS3, CP, NCP, and NSvc4, and that two peptides per target protein were mixed. Polyclonal antibodies were raised in rabbits according to instruction of AbFrontier company (Young In Frontier, Seoul, Korea). The detailed protocol for antigen preparation can be found in website of AbFrontier (https://www.abfrontier.com).

Protein extraction from plant tissues. Leaves of RSV infected plants (rice and weeds) from field were harvested and were kept in $-80{ }^{\circ} \mathrm{C}$. About $0.2 \mathrm{~g}$ of frozen plant materials were homogenized using a mortar and pestle. One milliliter of grinding buffer (50 mM Tris-HCl, $\mathrm{pH} 8.0,1$ mM EDTA) was applied to the samples and was ground continually. To extract proteins, we added $0.5 \mathrm{~mL}$ of $2 \mathrm{X}$ Laemili's loading buffer (4\% SDS, 20\% glycerol, 10\% 2- 
mercaptoethanol, $0.004 \%$ bromphenol blue, $0.125 \mathrm{M}$ Tris$\mathrm{HCl}, 0.2 \mathrm{M}$ dithiothreitol). Homogenized samples were boiled at $100{ }^{\circ} \mathrm{C}$ for $5 \mathrm{~min}$. And denatured samples were centrifuged at 13,000 rpm for $3 \mathrm{~min}$ using Gyro1524M. After centrifugation, the supernatant was transferred into a $1.5 \mathrm{~mL}$ tube and deposited at $-20^{\circ} \mathrm{C}$.

Western blot analysis. In advance to load protein samples in SDS-PAGE gel, proteins were boiled at $100{ }^{\circ} \mathrm{C}$ for 5 min and were resuspended in $30 \mu \mathrm{L}$ of Laemmli loading buffer (Laemmli, 1970), electrophoresed on 12\% SDS-polyacrylamide gel, and blotted onto PVDF transfer membranes (GE Healthcare, NJ, USA) (Towbin et al., 1992). The blots were probed with antiserum prepared against each His-tagged RSV protein or synthetic polypeptide. The pro- ducts were visualized using a NBT-BCIP solution (SigmaAldrich, Munich, Germany).

\section{Results and Discussion}

Generation of recombinant proteins for NS3 and CP proteins in $\boldsymbol{E}$. coli. In order to generate antibodies to detect RSV infection, we first selected four functionally important proteins with small protein size, such as NS3 and CP proteins from RNA3 segment, and NCP and NSvc4 from RNA4 segment (Table 1). The protein size of four proteins ranged from 21.5 to $35.1 \mathrm{kDa}$. For the generation of antibodies, we used two different approaches. The first is to use recombinant proteins which are generated by cDNA cloning and protein expression in $E$. coli followed by

Table 1. The list of RSV proteins used for antibody production.

\begin{tabular}{llll}
\hline \hline & \multicolumn{1}{c}{ NS3 } & CP \\
\hline Function & Gene silencing suppressor & Coat protein & Pathogenesis related protein \\
MW & $23.9 \mathrm{kDa}$ & $35.1 \mathrm{kDa}$ & $21.5 \mathrm{kDa}$ \\
Length & $636 \mathrm{bp}$ & $969 \mathrm{bp}$ & $536 \mathrm{bp}$ \\
Protein antiserum & Produced with negative result $(-)$ & Not developed & Produced with positive result $(+)$ \\
Peptide antiserum & Produced with negative result $(-)$ & Produced with positive result $(+)$ & Produced with positive result $(+)$ \\
\hline
\end{tabular}

Table 2. List of RSV peptides identified by bioinformatic program for antibody production. Of predicted peptides, two peptides for each protein were applied for antigen production

\begin{tabular}{|c|c|c|c|c|c|}
\hline Protein & No. & Peptide sequences & Position $^{\mathrm{a}}$ & Length & Antibody $^{b}$ \\
\hline \multirow{6}{*}{ NCP } & 1 & TLADLQKAINDISKD-C & $8-22$ & 16 & \\
\hline & 2 & KDKGGDTLAKDMTM-C & $56-70$ & 15 & \\
\hline & 3 & FVRDVTKKVKVAAGS-C & $81-95$ & 16 & \\
\hline & 4 & C-KKTMPEGKKKERGLTK & $199-214$ & 17 & \\
\hline & 5 & C-KKKEDGRKKVLDEF & $237-251$ & 16 & $\mathrm{O}$ \\
\hline & 6 & C-LVKEFFEDEAEGADD & $308-322$ & 16 & $\mathrm{O}$ \\
\hline \multirow{5}{*}{ NS3 } & 1 & MNVFTSSVGSVEFD-C & $1-14$ & 15 & \\
\hline & 2 & C-YIYDIHSSRHPSIDEHQ & $41-58$ & 18 & \\
\hline & 3 & C-SHDKNLPEEYRLPT & $84-97$ & 15 & $\mathrm{O}$ \\
\hline & 4 & C-DNRKTLYRSPSKKRHK & $162-177$ & 17 & $\mathrm{O}$ \\
\hline & 5 & C-KYVDSSALEPSPGSSP & $193-208$ & 17 & \\
\hline \multirow{5}{*}{$\mathrm{CP}$} & 1 & MQDVQRTIEVSVGP-C & $1-14$ & 15 & \\
\hline & 2 & C-PDLKDPERVTEDTKK & $38-52$ & 16 & $\mathrm{O}$ \\
\hline & 3 & GGEDDDNALIDIG & $93-105$ & 13 & \\
\hline & 4 & KNFLRHPNRMSKDQIK-C & $121-136$ & 17 & \\
\hline & 5 & C-KAESSDTEEYEKVWKK & $147-162$ & 17 & $\mathrm{O}$ \\
\hline \multirow{5}{*}{ NSve4 } & 1 & C-DLSEESQKRVDNKNRK & $18-33$ & 17 & $\mathrm{O}$ \\
\hline & 2 & KQDYSVKAHRKATFN-C & $77-91$ & 16 & \\
\hline & 3 & KSYVNSSDQVEVEVR-C & $136-150$ & 16 & \\
\hline & 4 & C-EFEPLEDKAINHLSS & $218-232$ & 16 & \\
\hline & 5 & C-KQPKKIPITKKSKSEVS & $267-283$ & 18 & $\mathrm{O}$ \\
\hline
\end{tabular}

${ }^{a}$ Position indicates the first and last amino acid covering the sequence in each viral protein.

${ }^{\text {b}}$ Peptides used for antibody production. 
A

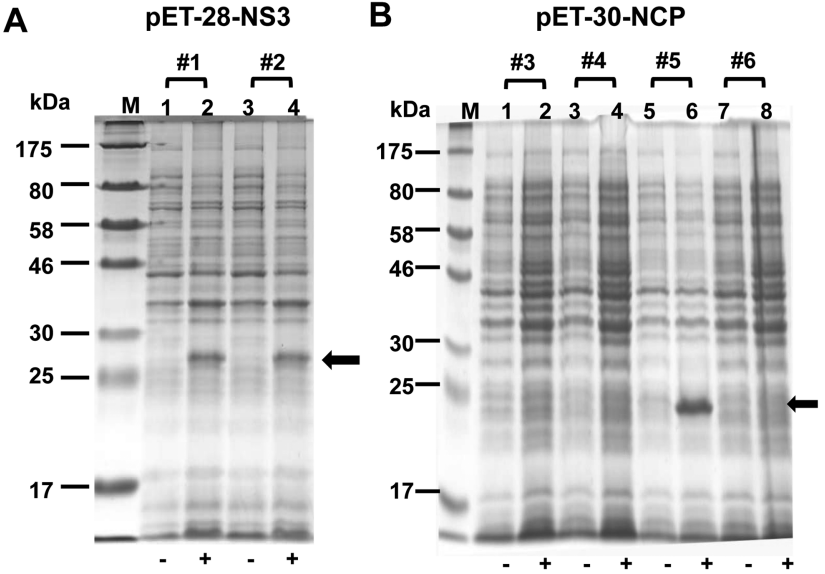

Fig. 1. SDS-PAGE analysis of the cell lysates derived from induced host bacteria $E$. coli BL21 carrying the recombinant expression vector. (A) Lysates of E. coli BL21 carrying pET-28NS3 without IPTG $(-)$ for lane 1 and 3 , and with IPTG $(+)$ for lane 2 and 4 . The \#1 and \#2 indicate two independent cells carrying the pET-28-NS3 construct. (B) Lysates of E. coli BL21 carrying pET30-NCP without IPTG (-) for lane 1, 3, 5, and 7, and with IPTG (+) for lane 2, 4, 6, and 8. The \#3, \#4, \#5, and \#6 indicate four independent cells carrying the pET-30-NCP construct. M represents protein marker and black arrows indicate expected bands of recombinant proteins.

protein purification. The other is to use synthetic polypeptides for protein of interest.

For the production of recombinant proteins, we amplified genes encoding NCP and NS3 proteins by RT-PCR and purified PCR products were cloned into pET-28 and pET30 expression vector, respectively. Two viral proteins fused with His-tag were expressed in E. coli BL21. To select proper bacterial cells expressing target proteins, we performed SDS-PAGE analysis separating cell lysates which were expressed in individual bacterial cell carrying the expression vector. From two independent bacterial cells, we could detect an additional band about $24-28 \mathrm{kDa}$ with presence of IPTG (Isopropyl $\beta$-D-1-thiogalactopyranoside) (Fig. 1A). In case of NCP-His-tag, we found an additional protein band from \#5 cell with IPTG (Fig. 1B). The size of protein band was almost similar to that of expected size (about $22 \mathrm{kDa}$ ). After recombinant protein purification using the lysate \#5 in lane 6 (Fig. 1B), start and flow-through materials from NS3-His-tag showed a large number of protein bands (Fig. 2). After washing, the first eluate includes few proteins with low amounts. Among six eluates, the second eluate (E2) contains significant amounts of multiple additional proteins while other eluates (E3-E6) have almost a specific band (Fig. 2). In final, we used the sixth eluate (E6) in which most of non-specific proteins were removed for immunization.

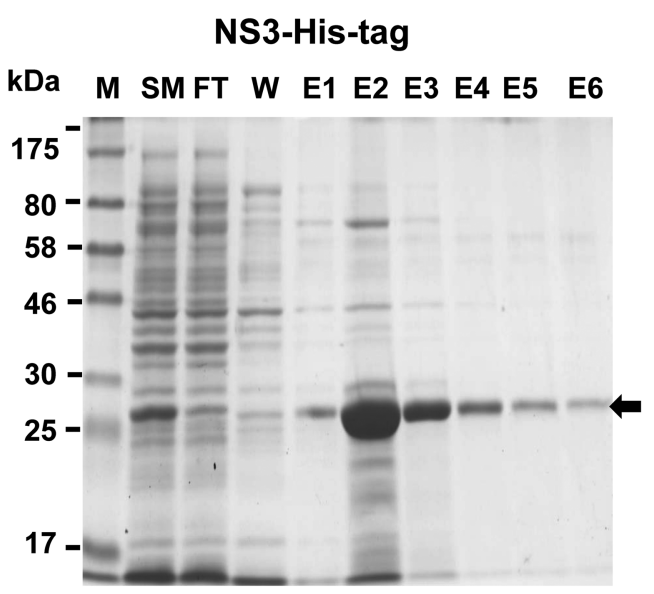

Fig. 2. SDS-PAGE analysis of purified His-tagged recombinant proteins using His GraviTrap ${ }^{\mathrm{TM}}$ columns. SDS-PAGE showed purified recombinant proteins for NS3-His-tag after expression in E. coli BL21. M, protein marker; SM, start material; FT, flowthrough; W, washed material; E1-E6, eluates. Black arrow indicates an expected band of recombinant protein.

Assessment of generated antibodies to detect RSV in RSV infected rice and weeds. For the recombinant protein-antibody, we used NCP and NS3 fused with His-tag. However, we obtained consistent positive results from antiNCP antibody only (data not shown). To test the anti-NCP antibody to detect RSV, we collected plant materials including rice and weeds which were infected by RSV. RTPCR was performed to confirm whether the collected samples have been infected with RSV (Fig. 3A and 3B). We used RSV infected samples from two different regions for western blot analysis whether newly developed RSV antibodies can be applied for RSV detection regardless of their genotypes. RT-PCR results confirmed RSV infections except samples 1 and 5, all rice samples were infected by RSV (Fig. 3A). Western blot analysis identified bands with $21.5 \mathrm{kDa}$ in rice sample 6 and 8 (Fig. 3C). Unfortunately, we could not detect any bands from samples 1-5 whereas sample 6 and 8 showed very strong bands. Although we loaded equal amount of proteins, we observed the differences in RSV infection severity from semi-quantitative RT-PCR (Fig. 3A and 3B) and thus the amount of viral proteins loaded in each lane might not be identical resulting in unsuccessful identification of virus infection. In addition, disease severity and/or infection time caused by RSV isolates of each collected sample might contribute to the production of individual RSV protein. Because we collected RSV infected plant leaves based on the symptoms but did not know the exact infection time and the relative amount of target RSV proteins.

Although it is well known that rice is the major host for RSV, it can also infect other plants such as weeds. To test 
A

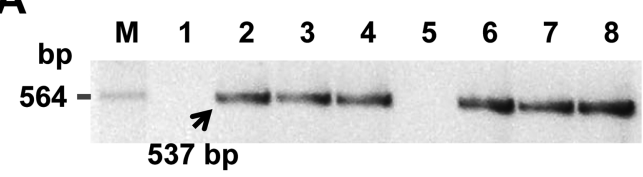

B

C

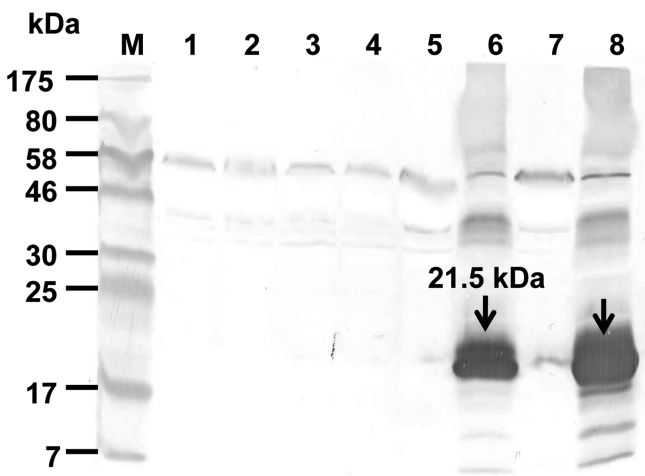

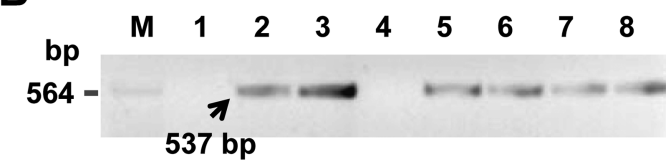

D

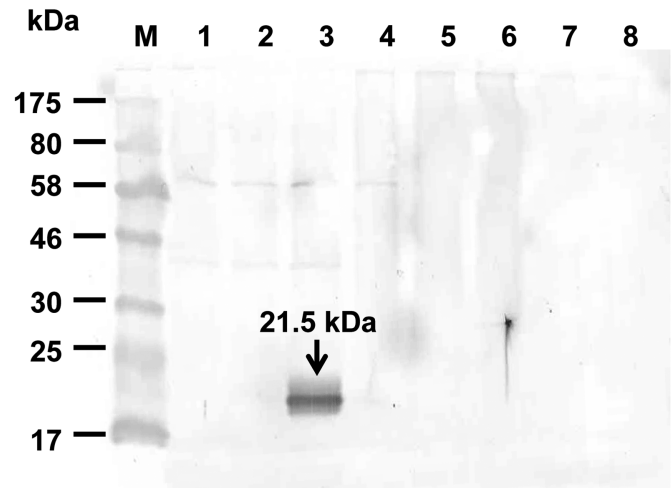

Fig. 3. RT-PCR and Western blot analysis to detect Rice strip virus (RSV) using recombinant protein-antibodies. (A \& B) RT-PCR detection of RSV RNA. Accumulation of RSV RNA was determined by RT-PCR as described in the Materials and Methods. Except samples 1 and 5 in panel A, 1 and 4 in panel B, and all samples were positive to RSV by RT-PCR detection. (C \& D) Western blot analysis. Proteins extracted from RSV infected rice and weeds were separated on SDS-PAGE gel. After blotting on the PVDF membrane, two protein-antibodies were applied for western blot analysis. RSV detection in RSV-infected rice (C) and weeds (D) by western blot analyses using anti-NCP antibody derived from the His-tag recombinant protein. (C) The rice samples were collected from Kimje (lanes 1-4) and Seocheon (lanes 5-8). (D) The weeds samples were collected from Anheung (green bristle grass; Setaria viridis; lanes 1-3 and crabgrass; Digitaria sanguinalis; lanes 4-6) and Seosan (barnyardgrass; Echinochloa crus-galli; lanes 7-8) and used for western blot analysis. Anti-NCP antibody was diluted to 1:20000.

whether generated anti-NCP antibody can be also useful to detect RSV in RSV infected weeds, we collected three different kinds of weeds like green bristle grass (Setaria viridis), crabgrass (Digitaria sanguinalis), and barnyardgrass (Echinochloa crus-galli). Of three RSV infected weeds, only green bristle grass showed a clear band about $21.5 \mathrm{kDa}$ (Fig. 3D). This result suggests that the generated anti-NCP can be implicated in detection of RSV at least in rice and green bristle grass and also suggest that there is a sensitivity difference between RT-PCR and antibody based detection methods as previously reported.

In addition to antibodies generated from recombinant proteins, we also tested antisera derived from synthetic peptides. A total of four antisera for NCP, CP, NS3, and NSvc4 were used for western analysis (Table 1). To optimize titer of each antiserum, we first tested individual antibody with various titers. Finally, optimal titer was obtained such as 1:50,000 titer for NCP, 1:10,000 titer for CP, 1:1,000 titer for NS3, and 1:1,000 titer for NSvc4. To compare efficiency of each antibody, we isolated proteins from RSV infected rice and two kinds of weeds like green bristle grass and crabgrass. Extracted proteins were separated on SDSPAGE and visualized by staining with Commassie Brilliant Blue (Fig. 4A). Although same amount of proteins were loaded, the band pattern was totally different from each other. For example, proteins in lanes 1-4 were extracted from rice, however, lanes 1 and 3 showed similar banding pattern with strong bands about $55 \mathrm{kDa}$ which might be the large subunit of Rubisco, unassociated with RSV infection, whereas lanes 2 and 4 displayed two strong bands about 35 $\mathrm{kDa}$ and $25 \mathrm{kDa}$ imposing that these two additional bands might also be derived from RSV infection (Fig. 4A). Lanes 5-8 for weed proteins showed relatively weaker bands as compared to rice proteins (Fig. 4A). Despite of the differences in banding pattern, all proteins in lanes $1-8$ exhibited common bands of about $28 \mathrm{kDa}$ and $55 \mathrm{kDa}$ (Fig. 4A).

Using a antisera for NCP and $\mathrm{CP}$, we detected $\mathrm{NCP}$ and $\mathrm{CP}$ proteins from three samples including two rice and one green bristle grass (Fig. 4B and 4C). Based on the intensity of band, rice samples possessed higher RSV concentration than that of green bristle grass. Antisera for CP and NS3 revealed RSV only from rice samples (Fig. 4D). Especially, the detected band size (about $28 \mathrm{kDa}$ ) for anti-NS3 derived from synthetic peptides was slightly higher as compared to the expected band size (about $23.9 \mathrm{kDa}$ ) (Fig. 4D). This result is highly correlated with the result from purified Histagged NS3 protein (Fig. 2). The increase of protein size may result from post-translational modification, or unusual amino acid composition leading to anomalous migration. Anti-NSvc4 antibody detected only RSV protein in one rice 


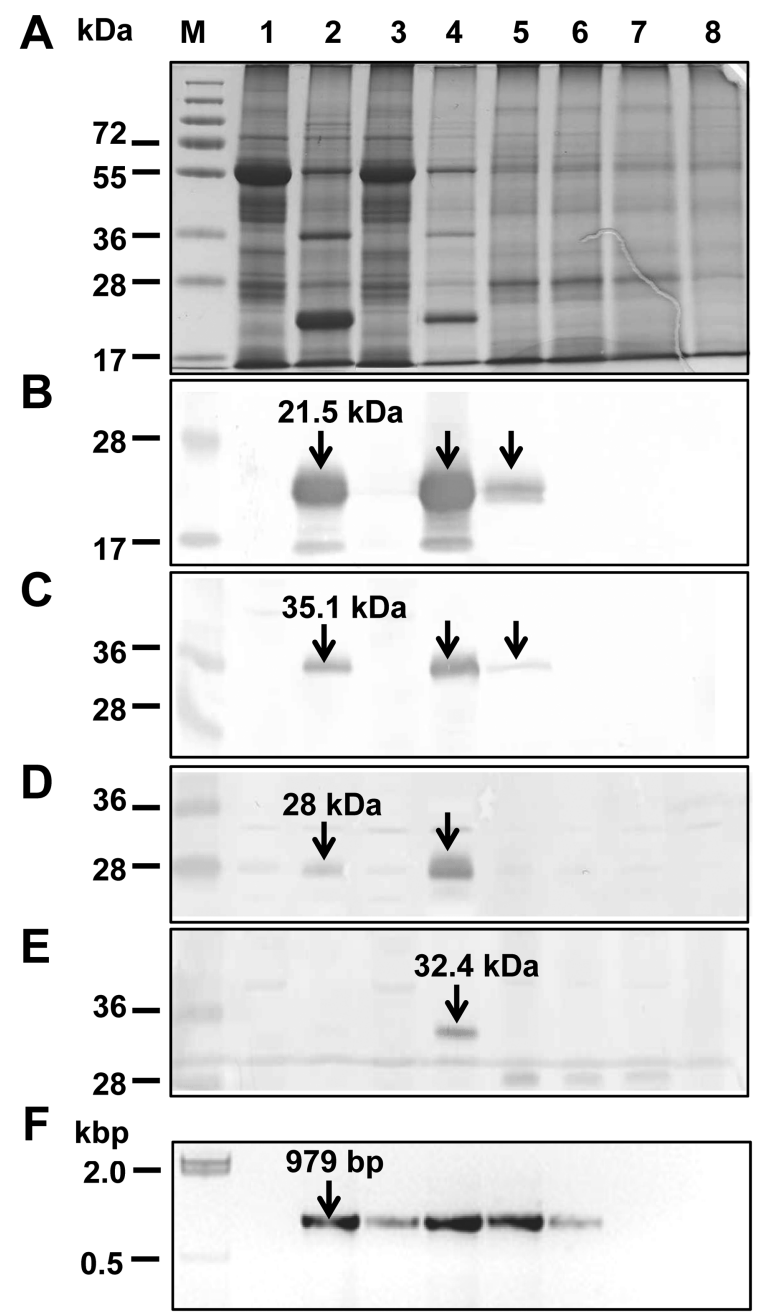

Fig. 4. Western blot analysis to detect RSV using peptide-antibodies. Proteins extracted from RSV infected rice and weeds were separated on SDS-PAGE gel. To visualize separated proteins, SDSPAGE gel was stained with Commasie Brilliant Blue (A). The blot on PVDF membrane was probed with peptide-antibody for NCP (B), CP (C), NS3 (D), and NSvc4 (E) proteins, respectively. Individual RSV viral protein was detected after reaction with freshly prepared NBT-BCIP substrate. M: Molecular marker. Lanes 1-4, rice samples collected from Seocheon region; Lanes 5-7, green bristle grass (Setaria viridis) samples collected from Anheung region; and Lane 8, crabgrass (Digitaria Sanguinalis) samples collected from Anheung region. Antibody dilution rates for NCP, $\mathrm{CP}, \mathrm{NS} 3$, and NSvc4 proteins were 1: 50,000 titer, 1: 10,000 titer, 1: 1,000 titer, and 1: 1,000 titer, respectively. (F) RT-PCR detection of RSV RNA. Accumulation of RSV RNA was determined by RT-PCR as described in the Materials and Methods. Except samples loaded in lanes 1, 7 and 8 , all samples were positive to RSV by RT-PCR detection.

sample (Fig. 4E). As shown in RT-PCR results, the amount of RSV RNAs seems to be highly correlated with that of RSV proteins (Fig. 4F). Although we used the same SDSPAGE for western blot analysis, the obtained intensity for each protein band varied. This might be due to different titer of antibody. Of four antibodies produced from synthetic peptides, anti-NCP seems to be superior with reliable protein detection ability including rice and green bristle grass.

In this study, we generated antibodies for RSV proteins using recombinant proteins and synthetic peptides as antigens. So far, two groups have developed monoclonal or polyclonal antibodies against RSV in our knowledge (Omura et al., 1986; Liang et al., 2005). The monoclonal antibody against RSV was produced in mice using the hybridoma cells (Omura et al., 1986). The developed antibody was evaluated for detection of RSV in infected plants and viruliferous insects using latex flocculation tests and enzymelinked immunoosorbent assay (ELISA). However, this antibody can recognize the whole RSV structure and not the individual RSV viral proteins. To overcome such limitation, a research group recently developed RSV antibodies against all RSV proteins except RdRp in RNA1 using recombinant proteins as antigens (Liang et al., 2005). They also observed that the size of NSvc4 body was increased than expected while other antibodies showed the expected sizes. Similarly, our results are consistent with their report indicating that protein detection was dependent on the time of RSV infection and/or the relative amount of each target protein. For instance, NCP antibody could detect the protein from early (3 weeks) and late (more than 4 months) infection. In contrast, NSvc4 antibody could detect infected leaves at early stage of infection (Liang et al., 2005). Therefore, we assume that accumulation of individual RSV protein in infected leaves might be variable and this might be the reason why some antibodies failed to detect the proteins in infected leaves in this study.

Most of antibodies that were produced using recombinant proteins require several different procedures such as cloning and purification. As compared to recombinant protein based approach, synthetic peptides have many advantages. For instance, as we have shown in this study, it does not need cloning and purification steps. Most important step is the selection of proper peptides which represent the target viral protein. Moreover, antigens using synthetic peptides produced more effective antibodies with high rate of success and specificity. However, synthetic peptides have also weak points such as lower affinity and longer immunization time as compared to recombinant proteins. Regardless of such disadvantages, our study showed that antibodies produced from synthetic peptides were superior to those generated from recombinant proteins.

\section{Acknowledgements}

This research was supported in part by grants from the Korea Institute of Planning and Evaluation for Technology of Food, Agriculture, Forestry and Fisheries (Nos. 108103- 
02 \& 309015-04) and the Rural Development Administration (No. PJ006952). SL, MGJ, and WKC were supported by research fellowships from the MEST through the Brain Korea 21 Project.

\section{References}

Abo, M. E. and Sy, A. A. 1998. Rice virus diseases: Epidemiology and management strategies. J. Sustain. Agr. 11:113-134.

Hamamatsu, C., Toriyama, S., Toyoda, T. and Ishihama, A. 1993. Ambisense coding strategy of the Rice stripe virus genome: in vitro translation studies. J. Gen. Virol. 74:1125-1131.

Hibino, H. 1996. Biology and epidemiology of rice viruses. Annu. Rev. Phytopathol. 34:249-274.

Jonson, M. G, Choi, H.-S., Kim, J.-S., Choi, I.-R. and Kim, K.-H. 2009a. Complete genome sequence of the RNAs 3 and 4 segments of Rice stripe virus isolates in Korea and their phylogenetic relationships with Japan and China isolates. Plant Pathol. J. 25:142-150.

Jonson, M. G, Choi, H.-S., Kim, J.-S., Choi, I. -R. and Kim, K.-H. 2009b. Sequence and phylogenetic analysis of the RNA1 and RNA2 segments of Korean Rice stripe virus isolates and comparison with those of China and Japan. Arch. Virol. 154:17051708.

Kisimoto, R. 1967. Genetic variation in the ability of a planthopper vector; Laodelphax striatellus (Fallen) to acquire the Rice stripe virus. Virology 32:144-152.

Laemmli, U. K. 1970. Cleavage of structural proteins during the assembly of the head of bacteriophage T4. Nature 227:680685.

Liang, D., Qu, Z., Ma, X. and Hull, R. 2005. Detection and localization of Rice stripe virus gene products in vivo. Virus Genes 31:211-221.
Maeda, H., Matsushita, K., Iida, S. and Sunohara, Y. 2006. Characterization of two QTLs controlling resistance to Rice stripe virus detected in a Japanese upland rice line, Kanto 72. Breed. Sci. 56:359-364.

Omura, T., Takahashi, Y., Shohara, K., Minobe, Y., Tsuchizaki, T. and Nozu, Y. 1986. Production of monoclonal antibodies against Rice stripe virus for the detection of virus antigen in infected plants and viruliferous insects. Ann. Phytopath. Soc. Japan 52:270-277.

Toriyama, S. 1986. Rice stripe virus: prototype of a new group of viruses that replicate in plants and insects. Microbiol. Sci. 3:347-351.

Toriyama, S. 2000. Rice stripe virus. CMI/AAB Description of Plant Viruses. No. 375.

Towbin, H., Staehelin, T. and Gordon, J. 1979. Electrophoretic transfer of proteins from polyacrylamide gels to nitrocellulose sheets: procedure and some applications. Biotechnology 24:145-149.

Wei, T. Y., Yang, J. G., Liao, F. L., Gao, F. L., Lu, L. M., Zhang, X. T., Li, F., Wu, Z. J., Lin, Q. Y., Xie, L. H. and Lin, H. X. 2009. Genetic diversity and population structure of Rice stripe virus in China. J. Gen. Virol. 90:1025-1034.

Xiong, R., Wu, J., Zhou, Y. and Zhou, X. 2008. Identification of a movement protein of the tenuivirus Rice stripe virus. J. Virol. 82:12304-12311.

Xiong, R., Wu, J., Zhou, Y. and Zhou, X. 2009. Characterization and subcellular localization of an RNA silencing suppressor encoded by Rice stripe tenuivirus. Virology 387:29-40.

Zhang, F., Guo, H., Zheng, H., Zhou, T., Zhou., Y, Wang S., Fang, R., Qian, W. and Chen, X. 2010. Massively parallel pyrosequencing-based transcriptome analyses of small brown planthopper (Laodelphax striatellus), a vector insect transmitting Rice stripe virus. BMC Genomics 11:303. 